\title{
The rearrangement process in a two-stage broadcast switching network
}

Jacobsen, Søren B.

Published in:

I E E E Transactions on Communications

Link to article, DOI:

$10.1109 / 26.2774$

Publication date:

1988

Document Version

Publisher's PDF, also known as Version of record

Link back to DTU Orbit

Citation (APA):

Jacobsen, S. B. (1988). The rearrangement process in a two-stage broadcast switching network. I E E E Transactions on Communications, 36(4), 484-491. https://doi.org/10.1109/26.2774

\section{General rights}

Copyright and moral rights for the publications made accessible in the public portal are retained by the authors and/or other copyright owners and it is a condition of accessing publications that users recognise and abide by the legal requirements associated with these rights.

- Users may download and print one copy of any publication from the public portal for the purpose of private study or research.

- You may not further distribute the material or use it for any profit-making activity or commercial gain

- You may freely distribute the URL identifying the publication in the public portal

If you believe that this document breaches copyright please contact us providing details, and we will remove access to the work immediately and investigate your claim. 


\title{
The Rearrangement Process in a Two-Stage Broadcast Switching Network
}

\author{
SOREN B. JACOBSEN
}

\begin{abstract}
This paper considers the rearrangement process in the twostage broadcast switching network presented by F. K. Hwang and G. W. Richards in IEEE TRANSACTIONS ON COMMUNICATIONS, October 1985. By defining a certain function it is possible to calculate an upper bound on the number of connections to be moved during a rearrangement. When each inlet channel appears twice, the maximum number of connections to be moved is found. For a special class of inlet assignment patterns in the case where each inlet channel appears three times, the maximum number of connections to be moved is also found. In the general case, an upper bound is given when the number of outlets at each second-stage switch is kept below a certain bound.
\end{abstract}

I. The KNown Properties of the Network

THE network to be considered here (see Fig. 1) is identical to the one presented in [1], and it is described by the three parametres $n_{1}, n_{2}$, and $M$ where

$n_{1}$ is the number of inlet channels at each first-stage switch,

$n_{2}$ is the number of outlets at each second-stage switch, and

$M$ is the number of times each inlet channel appears in the first stage.

The number of crosspoints in the network divided by the number of crosspoints in the corresponding rectangular switch is called the reduced number of crosspoints and is given by

$$
C_{\text {red }}=M\left(1 / n_{1}+1 / n_{2}\right) \text {. }
$$

To minimize $C_{\text {red }}$, the fraction $M / n_{2}$ has to be made as close to zero as possible but the rearrangement requirement puts a lower bound on the fraction.

Hall's theorem on a system of distinct representatives [2] ensures that the network is rearrangeable, if and only if, the following condition is fulfilled.

The Rearrangement Condition: For any $n \leq n_{2}$, there are at least $n$ first-stage switches containing appearance of any $n$ inlet channels.

To ensure that the $\boldsymbol{n}_{1}^{2}$ inlet channels are effectively rotated in the $M$ blocks the following condition is assumed to be fulfilled.

The Pair Condition: No pair of inlet channels appears on the same first-stage switch more than once throughout the $M n_{1}$ first-stage switches.

All the inlet assignment patterns presented in [1] fulfill the pair condition, but instead of working with some explicit patterns, it is more advantageous in a general approach just to assume the pair condition to be fulfilled.

II. AN UPPER BOUND ON THE NumBer OF CONNECTIONS TO BE Moved During a RearRangement by Means of THF FUNCTION $S_{M, n_{1}}$

As it will be seen later, the rearrangement condition as well as an upper bound on the number of connections to be moved

Paper approved by the Editor for Communication Switching of the IEEE Communications Society. Manuscript received November 19, 1986; revised July 2, 1987

The author is with the Electromagnetics Institute, Technical University of Denmark, DK-2800 Lyngby, Denmark.

IEEE Log Number 8718999.



Fig. 1. The network presented in [1]. Every inlet channel has exactly one appearance in each of the $M$ blocks.

during a rearrangement can be given by means of a function whose values are in general unknown. The function will be called $S_{M, n}$, and it is defined by means of another function $T_{M, n_{1}}$. Let $E$ be a subset of the set of inlet channels. Then,

$T_{M, n_{1}}(E):=$ The number of first-stage switches having elements from $E$ amongst its inlet channels.

Now $S_{M, n_{1}}$ is defined by

$$
S_{M, n_{1}}(n):=\min \left\{T_{M, n_{1}}(E) \mid E \text { has } n \text { elements }\right\} .
$$

$S_{M, n_{1}}$ is an increasing function, it depends on the inlet assignment pattern chosen, and $S_{M, n_{1}}(n)$ denotes the smallest number of first-stage switches that $n$ inlet channels can appear on.

In terms of $S_{M, n_{1}}$, we have

The network is rearrangeable if and only if

$$
n \leq S_{M, n_{1}}(n) \text { for all } n \leq n_{2} \text {. }
$$

This means that the optimal choice for $n_{2}$ is

$$
n_{2}=\max \left\{n \mid n \leq S_{M, n_{1}}(n)\right\} \text {. }
$$

An upper bound on the number of connections to be moved during a rearrangement can be found in terms of a sequence 
$\left\{s_{k}\right\}$ defined recursively from $S_{M, n_{1}}$ by

$$
s_{1}:=S_{M, n_{1}}(1) \text { and } s_{k+1}:=S_{M, n_{1}}\left(s_{k}+1\right) .
$$

The main result of this section is as follows.

Result 2.1: Let $r$ denote the integer with the property: $s_{r}$ $\leq n_{2}-1$ and $s_{r+1} \geq n_{2}$. Then the number of connections to be moved during a rearrangement will never exceed $r$.

Proof: To prove the result, consider the rearrangement algorithm given in [1]. It is characterized by the blocking relationship tree and its associated levels. The development of the tree stops at the first level where an idle switch arises. See Fig. 2. The important fact, given in [1], is

The number of connections to be moved is one less than the number of the level where the first idle switch arises.

Now the idea is to step through all levels of the blocking relationship tree, and in each level, keep an eye on the total number of inlet channels that have appeared so far, and to see how many first-stage switches they necessarily have appearance on. Sooner or later a level is reached where so many inlet channels have appeared, that the number of switches they demand, exeed the number of busy lines, which is, at most, $n_{2}$ - 1. When this occurs, at least one switch is idle.

The sequence $\left\{s_{k}\right\}$ is defined so that $s_{k}$ is a lower bound on the number of first-stage switches that have appeared in the first $k$ levels of the blocking relationship tree. This fact is explained in Fig. 3. Therefore, an idle switch must arise in the first level where $s_{k} \geq n_{2}$, which according to the definition of $r$ is level $r+1$. This concludes the proof of Result 2.1 .

To use Result 2.1 on a given network, it is sufficient to know $S_{M, n_{1}}(n)$ for $n=1,2, \cdots, n_{2}+1$. In the case $M=2$, $S_{M, n_{1}}$ is independent of $n_{1}$ and for $n \leq 6$ it takes the following values:

$$
\begin{array}{lr}
S_{2}(1)=2, \quad S_{2}(2)=3, \quad S_{2}(3)=4, & S_{2}(4)=4, \\
& S_{2}(5)=5, \quad S_{2}(6)=5 .
\end{array}
$$

This means that the optimal choice for $n_{2}$ is 5 , as given in [1] The sequence $\left\{s_{k}\right\}$ takes for $k \leq 3$, the values

$$
s_{1}=2, \quad s_{2}=S_{2}(3)=4, \quad s_{3}=S_{2}(5)=5=n_{2}
$$

from which we see that the integer $r$ defined in Result 2.1 equals 2 proving.

Result 2.2: The number of connections to be moved during a rearrangement will never exeed 2 , when $M=2$.

Fig. 2 shows a situation where two connections must be moved. This means that the maximum number of connections to be moved is two.

\section{The MaXimum Number of CONNections to Be MOVED During a Rearrangement fOR a SPECIAL Class OF INLET ASSIGNMENT PATTERNS WHEN $M=3$}

In this section, we consider the case $M=3$. Fix an inlet assignment pattern and let the switches in each of the 3 blocks be numbered $0,1, \cdots, n_{1}-1$. We will show that this inlet assignment pattern induces a latin square of order $n_{1}$. Define the $n_{1} \times n_{1}$ matrix $Z$ by

$i j$ 'te element in $Z$ is the number of the switch in block 3 , containing the common element of switch $i$ from block 1 and switch $j$ from block 2 .

This definition is taken from [3] and the pair condition ensures that $Z$ is a latin square. We restrict the calculation of $S_{3, n_{1}}$ to inlet assignment patterns where the induced latin square is the multiplication table of a group. Result 4.1 applied to the case
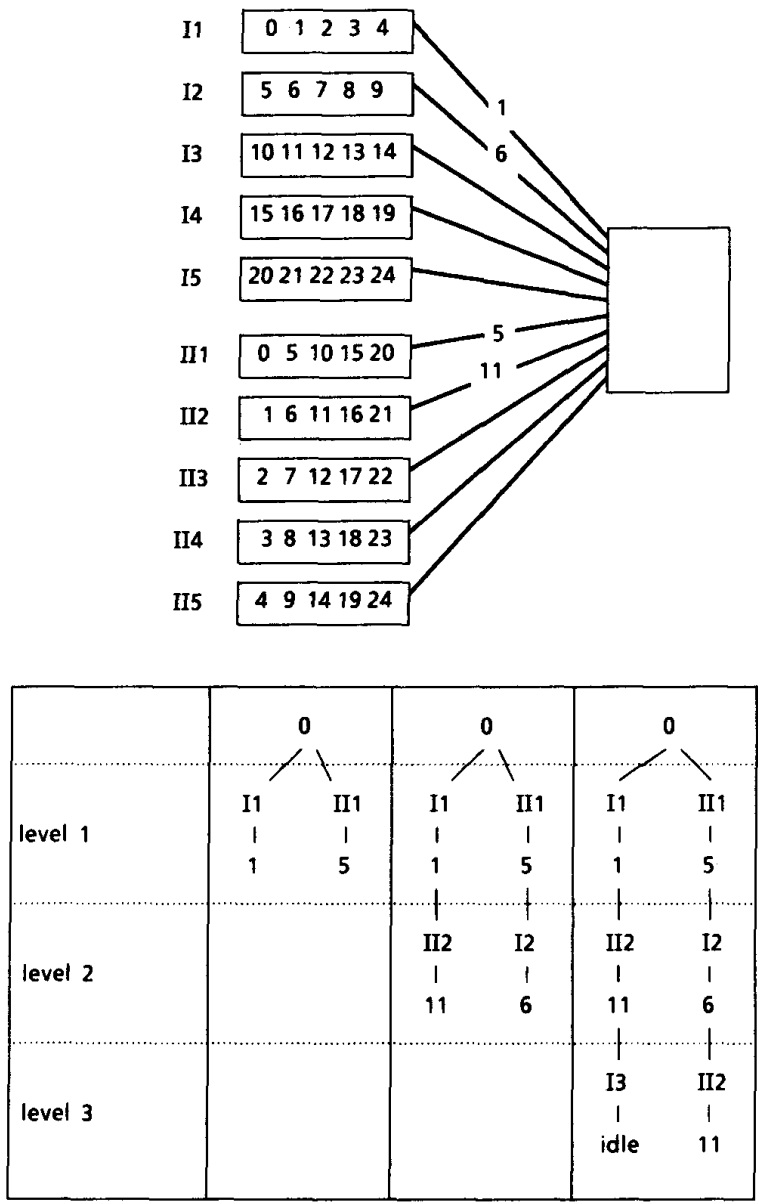

Fig. 2. The development of the blocking relationship tree. Here the tree stops in level 3 and the rearrangement is done by moving channel 11 to switch $\mathrm{I} 3$ and channel 1 to switch II2.

ao

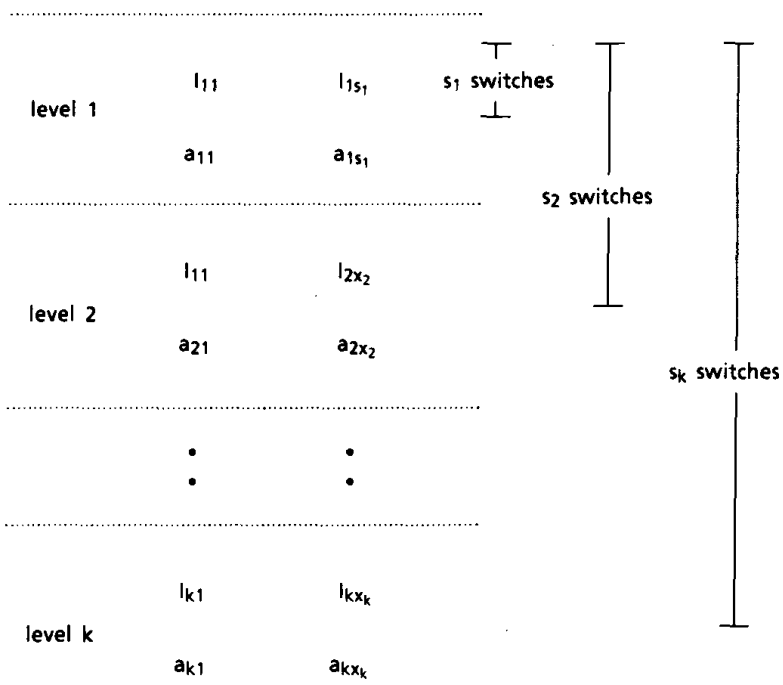

Fig. 3. Inlet channel $a_{0}$ is present at $s_{1}=S_{M, n_{1}}$ (1) first-stage switches. None of them are idle meaning that $s_{1}+1$ channels are present. They have appearance on at least $s_{2}=S_{M, n_{1}}\left(s_{1}+1\right)$ first-stage switches. If none of them are idle we now have a total of $s_{2}+1$ channels present, etc. 
$M=3$ together with the upper bound $U_{3}$ given in Appendix A yields

$$
\begin{array}{lll}
S_{3, n_{1}}(1)=3, & S_{3, n_{1}}(2)=5, & S_{3, n_{1}}(3)=6, \\
S_{3, n_{1}}(5)=8, & S_{3, n_{1}}(7)=9, & S_{3, n_{1}}(10)=11,
\end{array}
$$

$$
S_{3, n_{1}}(4)=6 \text { or } 7, \quad S_{3, n_{1}}(6)=8 \text { or } 9, \quad S_{3, n_{1}}(8)=9 \text { or } 10 \text {, }
$$$$
S_{3, n_{1}}(9)=9,10 \text { or } 11, S_{3, n_{1}}(11)=11 \text { or } 12 \text {, }
$$$$
S_{3, n_{1}}(13)=12 \text { or } 13 \quad S_{3, n_{1}}(14)=12 \text { or }^{1} 13 \text {. }
$$

We will now determine when $S_{3, n_{1}}(4)=6$. If $S_{3, n_{1}}(4)=6$ then there must exist four inlet channels appearing in two switches in block 1 , in two switches in block 2 , and in two switches in block 3 . (If all four inlet channels appeared on the same switch in one of the blocks then they would appear on four different switches in the remaining two blocks.) In the language of latin squares this means we can find four entries appearing in two rows and two columns so that these four entries contain only two different element which we call $x_{1}$ and $x_{2}$. Since the latin square corresponds to the multiplication table of a group, the two rows correspond to two group elements $a_{1}, a_{2}$, and the two columns correspond to two group elements $b_{1}, b_{2}$ so that the multiplication table for $\left\{a_{1}, a_{2}\right\} \times$ $\left\{b_{1}, b_{2}\right\}$ is

$$
\begin{array}{l|ll} 
& b_{1} & b_{2} \\
\hline a_{1} & x_{1} & x_{2} \\
a_{2} & x_{2} & x_{1}
\end{array}
$$

This yields $a_{1}=x_{1} b_{1}^{-1}$ and $a_{2}=x_{2} b_{1}^{-1}$ and therefore $x_{1} b_{1}^{-1} b_{2}$ $=x_{2}$ and $x_{2} b_{1}^{-1} b_{2}=x_{1}$ implying $\left(b_{1}^{-1} b_{2}\right)^{2}=1$. Since an element of order 2 exist, if and only if, 2 divides the order of the group, we have proved

$$
S_{3, n_{1}}(4)=\left\{\begin{array}{l}
6 \text { when } 2 \text { divides } n_{1} \\
7 \text { else }
\end{array}\right.
$$

In Appendix $\mathrm{A}$, similar methods are used to find $S_{3, n_{1}}(n)$ when $n=11$ and 13 . The remaining values can be calculated the same way. We therefore have the following.

Result 3.1: Assume $M=3$ and $n_{1} \geq 4$. For all inlet assignment patterns where the induced latin square is the multiplication table of a group, we have:

1) $S_{3, n_{1}}$ takes the values in Table $I$.

2) The optimal choice for $n_{2}$ (assuming $S_{3, n_{1}}(14) \leq 13$ for all inlet assignment patterns), and the maximum number of connections to be moved during a rearrangement are the numbers given in Table II.

When $n_{1}$ is a multiple of four or five, it is easy to construct states where a rearrangement requires 3 connections to be moved. Figs. 4 and 5 show a state where 4 (5) connections has to be moved. The upper bounds given in Table II are therefore the maximum number of connections to be moved.

Can the results in this section be extended so that they include arbitrary latin squares? The answer is no. Consider the following latin square:

$$
\begin{array}{lllll}
\frac{0}{1} & \frac{1}{0} & 2 & 3 & 4 \\
\frac{1}{2} & \frac{0}{4} & 3 & 4 & 2 \\
3 & 2 & 4 & 0 & 3 \\
4 & 3 & 1 & 2 & 0
\end{array} .
$$

\begin{tabular}{|c|c|}
\hline$n$ & $S_{3, n_{1}}(n)$ \\
\hline 1 & 3 \\
\hline 2 & 5 \\
\hline 3 & 6 \\
\hline 4 & $\begin{cases}6 & \text { when } 2 \text { divides } n_{1} \\
7 & \text { else }\end{cases}$ \\
\hline 5 & 8 \\
\hline 6 & $\begin{cases}8 & \text { when } 3 \text { divides } n_{1} \\
9 & \text { else }\end{cases}$ \\
\hline 7 & 9 \\
\hline 8 & $\left\{\begin{aligned} 9 & \text { when } 3 \text { divides } n_{1} \\
10 & \text { else }\end{aligned}\right.$ \\
\hline 9 & $\begin{cases}9 & \text { when } 3 \text { divides } n_{1} \\
10 & \text { when } 4 \text { but not } 3 \text { divides } n_{1} \\
11 & \text { else }\end{cases}$ \\
\hline 10 & 11 \\
\hline 11 & $\begin{cases}11 & \text { when } 4 \text { divides } n_{1} \\
12 & \text { else }\end{cases}$ \\
\hline 12 & $\begin{cases}11 & \text { when } 4 \text { divides } n_{1} \\
12 & \text { else }\end{cases}$ \\
\hline 13 & $\begin{cases}12 & \text { when } 4 \text { or } 5 \text { divides } n_{1} \\
13 & \text { else }\end{cases}$ \\
\hline
\end{tabular}

Select the four entries in the two upper rows and the two left columns and conclude that $S_{3,5}(4)=6$ in this case where 2 does not divides $n_{1}(=5)$.

${ }^{1}$ For latin squares corresponding to some special groups of order a power of 3 , it is in doubt whether $S_{3, n_{1}}(14)=13$ or 14. If $S_{3, n_{1}}(14)=14$ there exist inlet patterns where best $n_{2}$ is 14 .
TABLE I

THE VALUES OF THE FUNCTION $S_{3, n_{1}}$ FOR $1 \leqslant n \leqslant 13$

IV. AN UPPER BOUND ON THE NUMBER OF CONNECTIONS TO BE Moved DuRING a REARRANGEMENT WHEN $M$ IS ARBITRARY

When $n_{1}$ and $M$ grow, it becomes very time consuming to calculate the values of $S_{M, n_{1}}$. It would, therefore, be advantageous if upper and lower bounds could be given. In Appendix A we prove the following.

Result 4.1: For all inlet assignment patterns fulfilling the pair condition the following estimate is valid:

$$
S_{M, n_{1}}(n) \geq G_{M}(n) \text { for any } n \leq n_{1}^{2}
$$

where

$$
G_{M}(n)=\left\{\begin{array}{c}
(p+1) M-1 \text { when } p^{2}+1 \leq n \leq p^{2}+p \\
(p+1) M \text { when } p^{2}+p+1 \leq n \leq(p+1)^{2}
\end{array}\right.
$$

Since $G_{M}(n) \leq S_{M, n_{1}}(n)$, the network is rearrangeable as long as $n_{2} \leq \max \left\{n \mid n \leq G_{M}(n)\right\}$. But (4.2) gives that max $\left\{n \mid n \leq G_{M}(n)\right\}=M(M+1)-1$ so

$$
n_{2}=M(M+1)-1
$$

well known from [1].

To get an upper bound on the number of connections to be moved during a rearrangement the following sequence $\left\{g_{k}\right\}$ [compare to (2.4)] is defined

$$
g_{1}:=G_{M}(1) \text { and } g_{k+1}=G_{M}\left(g_{k}+1\right) \text {. }
$$

$g_{k}$ has the following two obvious properties:

1) $g_{k} \leq s_{k}$ for any $k$ and $g_{k}$ is therefore a lower bound on the number of first-stage switches that have appeared in the first $k$ levels of the blocking relationship tree.

2) Let $m$ be the integer with the property $g_{m} \leq n_{2}-1$ and $g_{m+1} \geq n_{2}$. Then $m$ is an upper bound on the number of connections to be moved during a rearrangement.

It is now easy to verify the following.

Result 4.2: If $n_{2} \leq M(M+1)-1$ then the number of 

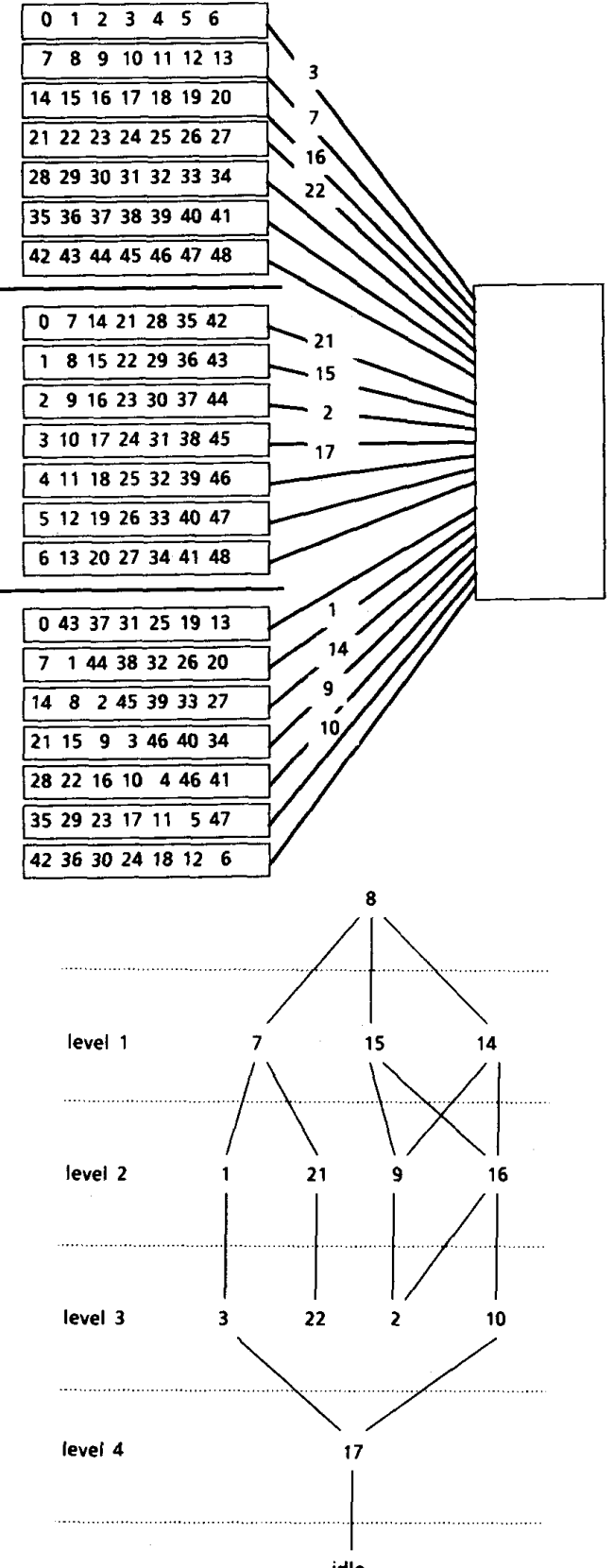

Fig. 4. In this network having a total of 49 inlet channels, a request is made for channel 8 and four connections have to be moved.

connections to be moved during a rearrangement will for $M \leq 21$ never exceed the numbers given in Table III.

In Appendix $\mathrm{C}$ it is proven that:

Result 4.3: If $n_{2} \leq M(M+1)-1$, then the number of connections to be moved during a rearrangement will never exceed $3+\left\lceil(1 / \ln 2) \ln \left(M^{2} \ln M\right)\right\rceil$ where $\lceil x\rceil$ is the smallest integer bigger than or equal to $x$.

Result 4.3 is not the best obtainable but it shows that the number of connections to be moved grow at most logarithmic in $\boldsymbol{M}$.

V. The Inlet Assignment Pattern and Finite Geometry

In this section, results from the theory of finite geometries is used to examine the inlet assignment pattern.
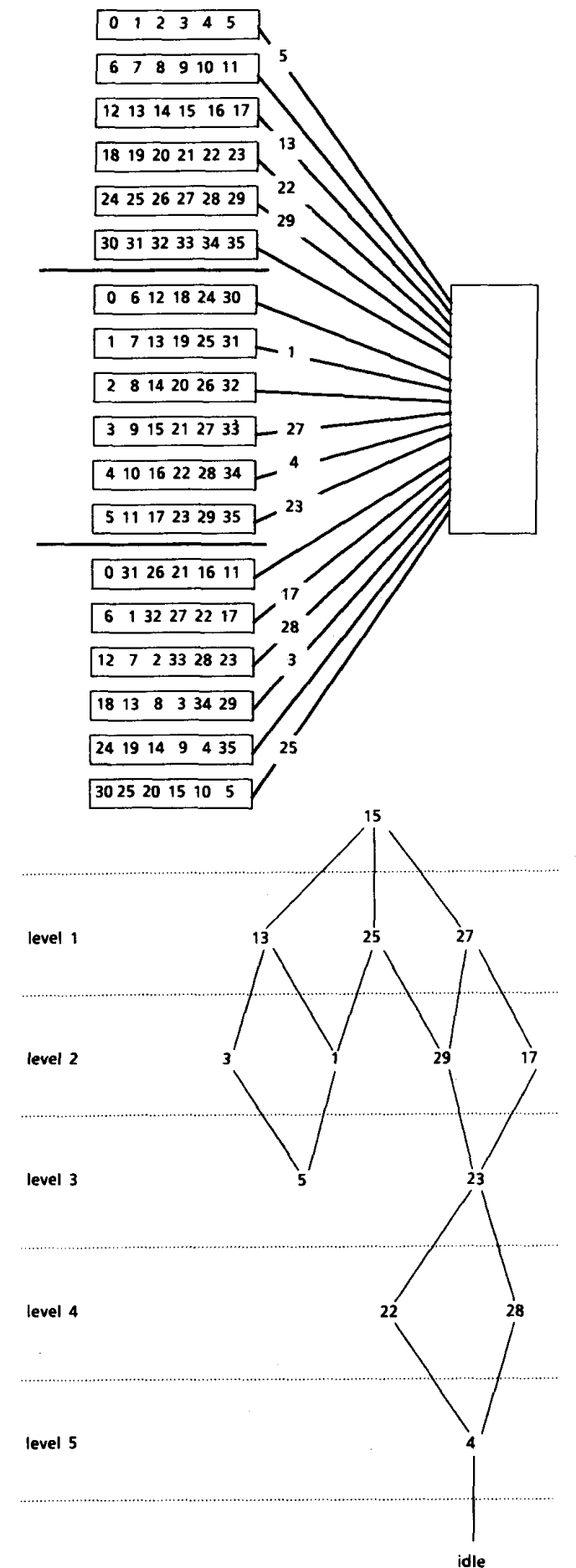

Fig. 5. In this network having a total of 36 inlet channels a request is made for channel 15 and five connections have to be moved.

According to the definition $[4$, p. 251], a geometric $k$ net is a set of points together with a set of lines appearing in $k$ different parallel classes such that

1) each point belongs to exactly one line of each parallel class

2) if $l_{1}$ and $l_{2}$ are lines of different parallel classes, then $l_{1}$ and $l_{2}$ have exactly one point in common

3) there are at least two points on each line. 
TABLE II

THE MAXIMUM NUMBER OF OUTLETS AT EACH SECOND STAGE SWITCH AND THE MAXIMUM NUMBER OF CONNECTIONS TO BE MOVED DURING A REARRANGEMENT

\begin{tabular}{|c|c|c|}
\hline & $\begin{array}{c}\text { Optimal value } \\
\text { for } n_{2}\end{array}$ & $\begin{array}{c}\text { The maximum number of } \\
\text { connections to be moved } \\
\text { during a rearrangement }\end{array}$ \\
\hline$n_{1}$ is a multiple of 4 & 11 & 3 \\
\hline$n_{1}$ is a multiple of 5 but not 4 & 12 & 3 \\
\hline$n_{1}$ is not a multiple of 4 or 5 & 13 & 5 \\
\hline$n_{1}$ is not a multiple of 2,3 or 5 & 13 & 4 \\
\hline
\end{tabular}

TABLE III

AN UPPER BOUND ON THE NUMBER OF CONNECTIONS TO BE MOVED DURING A REARRANGEMENT WHEN $n_{2} \leqslant M(M+1)-1$

\begin{tabular}{|c|c|c|c|c|c|c|c|}
\hline & $M=2$ & $M=3$ & $M=4$ & $M=5$ & $M=6, \ldots, 9$ & $M=10, \ldots, 14$ & $M=15, \ldots, 21$ \\
\hline $\begin{array}{c}\text { the upper bound } \\
m\end{array}$ & 2 & 3 & 4 & 4 & 5 & 6 & 7 \\
\hline
\end{tabular}

Consider a network with $n_{1}, M$, and an inlet assignment pattern given. The $n_{1}^{2}$ inlet channels correspond to the set of points. Each of the $M$ blocks corresponds to a parallel class, and each switch in a block corresponds to a line in this parallel class. Since each inlet channel is present exactly once in each block, 1) is fulfilled. The pair condition ensures that 2) is fulfilled, and for $n_{1} \geq 2$ the inlet assignment pattern given is a geometric $M$-net of order $n_{1}$.

This connection to $k$ net can be used to find the highest possible value of $\boldsymbol{M}$ before the pair condition is violated. For $n_{1} \leq 9$, we have $([4, \mathrm{ch} .8])$.

The function $S_{M, n_{1}}$ is known in general to depend on the inlet assignment pattern chosen. To be more precise an equivalence relation is introduced. Let $\boldsymbol{P}_{1}$ and $\boldsymbol{P}_{2}$ be two inlet assignment patterns in the same network, i.e., $M$ and $n_{1}$ is fixed. Then,

$$
P_{1} \sim P_{2} \Leftrightarrow S_{M, n_{1}, P_{1}}=S_{M, n_{1}, P_{2}} .
$$

The equivalence relation splits the set of inlet assignment patterns for the network into classes and in order to obtain the best network an inlet pattern that makes $S_{M, n_{1}}$ as big as possible has to be chosen.

When $M=2$, there is for any $n_{1}$ only one class, and it is therefore impossible to improve the network by using inlet patterns different from the one used in the Fig. 2.

When $M=3$, the results in Section III prove that $S_{3, n_{1}}$ depends on $n_{1}$ and for $n_{1} \geq 5$ there are in general more than one class. From lemma 1 in [3], it can be seen that for $n_{1}$ a prime all inlet assignment patterns made from the subarrays given in [1] are contained in only one class.

For a general $M$ and $n_{1}$, it seems very difficult to determine the classes. Then it seems more practical to find an useful upper bound $U_{M}$ on $S_{M, n_{1}}$, which can be used to decide whether or not a given inlet pattern makes $S_{M, n_{1}}$ big enough. In [4] and [5], geometric nets are used to construct projective planes, and it is not unlikely that methods and results there can be helpful in finding an useful upper bound.

\section{CONCLUSIONS}

In this paper, an upper bound on the number of connections to be moved during a rearrangement in a two-stage broadcast switching network is found. In general, the bound is given in
TABLE IV

THE MAXIMUM NUMBER OF TIMES EACH INLET CHANNEL CAN APPEAR IN THE FIRST STAGE BEFORE THE PAIR CONDITION IS VIOLATED

\begin{tabular}{|l|l|l|l|l|l|l|l|l|l|}
\hline$n_{1}$ & 2 & 3 & 4 & 5 & 6 & 7 & 8 & 9 & $p^{n}$ \\
\hline $\begin{array}{c}\text { Highest } \\
\text { possible } \\
\text { value of } M\end{array}$ & 3 & 4 & 5 & 6 & 3 & 8 & 9 & 10 & $p^{n}+1$ \\
\hline
\end{tabular}

terms of the function $S_{M, n_{1}}$, which means that when the values of $S_{M, n_{1}}$ are known, then the upper bound is easily calculated.

When $M=2$ the function $S_{M, n_{1}}$ is independent of $n_{1}$ and of the inlet assignment pattern, and two is the maximum number of connections to be moved during a rearrangement.

When $M=3$ the function $S_{M, n_{1}}$ depends on $n_{1}$. For a special class of inlet patterns the values of $S_{3, n_{1}}$ is found, and the optimal choice for $n_{2}$ is 11 when $n_{1}$ is a multiple of 4 , it is 12 when $n_{1}$ is a multiple of 5 but not 4 , and it is 13 in the other cases. The maximum number of connections to be moved during a rearrangement is 3 when $n_{1}$ is a multiple of 4 or 5 . When $n_{1}$ is not a multiple of 4 or 5 , the maximum number of connections to be moved is 5 and when $n_{1}$ is a prime it is 4 .

In the case where $M$ is arbitrary the pair condition is used to find a lower bound $G_{M}$ on $S_{M, n_{1}}$, and this lower bound yields that the number of connections to be moved during a rearrangement grows, at most, logarithmic as a function of $M$ when the number of outlets at each second-stage switch is not exceeding $M(M+1)-1$.

Finally, the close connection between the inlet assignment pattern and finite geometry is considered.

\section{APPENDIX A}

The Calculations of $S_{3, n_{1}}$

We first find an upper bound $U_{3}$ for $S_{3, n_{1}}$. Let for $1 \leq n \leq$ 14, $U_{3}$ be defined by Table $\mathrm{V}$.

Result A.1: Assume $n_{1} \geq 4$. For all inlet assignment patterns where the induced latin square is the multiplication table of a group we have $S_{3, n_{1}}(n) \leq U_{3}(n), n=1,2$, $\cdots, 13 . S_{3, n_{1}}(14) \leq 13$ when the induced latin square 
TABLE $\mathrm{V}$

AN UPPER BOUND ON THE FUNCTION $S_{3, n_{1}}$ INDEPENDENT OF $n_{1}$

\begin{tabular}{|c|c|c|c|c|c|c|c|c|c|c|c|c|c|c|}
\hline$n$ & 1 & 2 & 3 & 4 & 5 & 6 & 7 & 8 & 9 & 10 & 11 & 12 & 13 & 14 \\
\hline$U_{3}(n)$ & 3 & 5 & 6 & 7 & 8 & 9 & 9 & 10 & 11 & 11 & 12 & 12 & 13 & 13 \\
\hline
\end{tabular}

corresponds to a group not isomorfic to groups of the form $Z_{3} \times \cdots \times Z_{3}$.

Proof: Assume $1 \leq n \leq 13$. By explicitly selecting the rows, columns, and entries in the latin square the way described in $[3$, Sect. II], we get the result when the latin square is the multiplication table of $Z_{n_{1}}$ (the cyclic group of order $n_{1}$ ). For the groups $Z_{2} \times Z_{2}, Z_{3} \times Z_{3}$, and $\Sigma_{3}$ (the permutation group of three elements), it is also easy by explicit selection to show that $U_{3}$ is upper bound in these cases.

To prove the result in the general case, assume that $G$ is an arbitrary finite group of order $n_{1}$. In the rest of the proof, we will use the result from the standard group theory, see for example, [6, chap. I.6]. Let $p$ denote the greatest prime that divides $n_{1}$. Then $G$ has an element of order $p$ and, therefore, a cyclic subgroup of order $p$. When $p \geq 5$, we get the result by choosing the rows, columns, and entries among the elements of this subgroup. If $n_{1}$ is a power of $2, G$ is a two-group and therefore it has a subgroup of order 4 , which is either $Z_{4}$ or $Z_{2}$ $\times Z_{2}$. Conclude by choosing rows, columns, and entries among this subgroup. When $n_{1}$ is a power of $3, G$ is a threegroup and therefore it contains a subgroup of order 9 (either $Z_{9}$ or $Z_{3} \times Z_{3}$ ). Finally, if $n_{1}=2^{k} 3^{l}$, then $G$ has a subgroup of order $2^{k}$ and a subgroup of order $3^{l}$ leaving only $n_{1}=6$ as unsolved. But $Z_{6}$ and $\Sigma_{3}$ are the only groups of order 6 and $U_{3}$ is known to be an upper bound in these cases.

For $n=14$ and the latin square equal to $Z_{3} \times Z_{3}$, the author has only been able to select 14 entries in a way that proves $S_{3, n_{1}, Z_{3} \times Z_{3}}(14) \leq 14$. When the latin square corresponds to a group of the form $Z_{3} \times \cdots \times Z_{3}$, it is therefore in doubt whether $S_{3, n_{1}}(14)=13$ or 14 .

Result 4.1 in the case $M=3$ and Result A.1 determine $S_{3, n_{1}}(n)$ when $n=1,2,3,5,7,10$. Since the calculation of $S_{3, n_{1}}$ proceeds the same way for $n=4,6,8,9,11,12,13,14$, we only consider the two most difficult cases $n=11$ and $n=$ 13 here. We start with the following.

Lemma A.1: Let $G$ be a finite group of order at least 4 . Let $I, a, b, c$ be four distinct elements of $G$ where 1 denotes the identity element. Assume that $a^{2}=1$. Consider the multiplication table for $\{1, a, b, c\}$. Put for $j=1,2,3,4$,

$C_{j}:=$ the set of elements in column $j$

of the multiplication table

\begin{tabular}{c|cccc} 
& 1 & $a$ & $b$ & $c$ \\
\hline 1 & 1 & $a$ & $b$ & $c$ \\
$a$ & $a$ & 1 & $a b$ & $a c$ \\
$b$ & $b$ & $b a$ & $b^{2}$ & $b c$ \\
$c$ & $c$ & $c a$ & $c b$ & $c^{2}$
\end{tabular}

If $\left|C_{1} \cap C_{j}\right| \geq 3$ for $j=2,3$ or $j=2,4$, then $G$ contains a subgroup of order 4.

Proof: Since $\left|C_{1} \cap C_{2}\right| \geq 3$, we must have $b a=c$ or $c a$ $=b$ since no element appears more than once in a row or a column. Since $a^{2}=1$ this implies that $b a=c$ and $c a=b$. The multiplication table then looks as follows:

\begin{tabular}{l|llll} 
& 1 & $a$ & $b$ & $c$ \\
\hline 1 & 1 & $a$ & $b$ & $c$ \\
$a$ & $a$ & 1 & - & - \\
$b$ & $b$ & $c$ & - & - \\
$c$ & $c$ & $b$ & - & -
\end{tabular}

We only prove the lemma in the case where $\left|C_{1} \cap C_{j}\right| \geq 3$ for $j=2,3$, Then there are three possibilities to consider: 1) $a b$ is the unknown element, i.e., we do not know whether or not $a b$ $\in\{1, a, b, c\}, 2) b^{2}$ is the unknown, or 3) $c b$ is the unknown element.

Assume that $a b$ is the unknown element: Then $b^{2}=a$ or $b^{2}$ $=1$. If $b^{2}=a$ then $b$ has order 4 and the subgroup generated by $b$ is a subgroup of order 4 . If $b^{2}=1$ we have $c b=a$ implying $c=c b^{2}=a b$. But then $a^{2}=1, b^{2}=1, c=a b=$ $b a$ and therefore $\{1, a, b, c\}$ is the Klein Four Group $\left(Z_{2} \times\right.$ $Z_{2}$ ).

Assume that $b^{2}$ is the unknown element; then $a b=c$. Multiplying by $a$ from left gives $b=a c$. Now $c b=1$ or $c b$ $=a$. If $c b=1$ then $a=a c b=b^{2}$ implying that $b$ has order four. If $c b=a$ then $1=a^{2}=a c b=b^{2}$ and then $a b=b a=$ $c$ implying that $\{1, a, b, c\}$ is the Klein Four Group.

Assume that $c b$ is the unknown element; then $a b=c$ and therefore $b^{2}=1$ or $b^{2}=a$. If $b^{2}=1,\{1, a, b, c\}$ is the Klein Four Group and if $b^{2}=a, b$ has order 4 . Since we have now covered all cases the proof of lemma A.1 is completed.

We now proceed with the calculation of $S_{3, n_{1}}$. The fact that no element appears more than once in a row (column) will be used without comment

$n=11$ : Assume $S_{3, n_{1}}(11)=11$. If the 11 inlet channels appeared on only two switches in one of the blocks then they would have to appear on at least six switches in each of the remaining two blocks and the 11 inlet channels would then appear on at least 14 switches. Because of the symmetry in rows and columns, we only have to consider the following two cases: 1) when the 11 inlet channels appear on four switches in block 1 , four switches in block 2 , and three switches in block $3 ; 2$ ) when the 11 channels appear on four switches in block 1 , three switches in block 2 , and four switches in block 3 .

Case 1): In the language of latin squares we have 11 entries containing only three different elements $\left(x_{1}, x_{2}, x_{3}\right)$ and appearing in four rows and four columns. The four rows (columns) correspond to four group elements $a_{1}, a_{2}, a_{3}, a_{4}\left(b_{1}\right.$, $\left.b_{2}, b_{3}, b_{4}\right)$. Since it is 11 entries containing only $x_{1}, x_{2}, x_{3}$, three of the rows and three of the columns contain all the elements $x_{1}, x_{2}, x_{3}$ and the remaining row and column contains two of these three elements. After possible renaming of the group elements we may assume that we have the following table:

\begin{tabular}{l|cccc} 
& $b_{1}$ & $b_{2}$ & $b_{3}$ & $b_{4}$ \\
\hline$a_{1}$ & $x_{1}$ & $x_{2}$ & $x_{3}$ & - \\
$a_{2}$ & $x_{2}$ & - & - & - \\
$a_{3}$ & $x_{3}$ & - & - & - \\
$a_{4}$ & - & - & - & -
\end{tabular}

where row 1,2 , and 3 and column 1,2 , and 3 or 4 , contain $x_{1}$, $x_{2}, x_{3}$ while row 4 and column 4 or 3 contain two of these three elements. Multiply the row elements by $a_{1}^{-1}$ from the left and the column elements by $b_{1}^{-1}$ from the right and obtain

\begin{tabular}{c|cccc} 
& 1 & $a$ & $b$ & $d$ \\
\hline 1 & 1 & $a$ & $b$ & $d$ \\
$a$ & $a$ & - & - & - \\
$b$ & $b$ & - & - & - \\
$c$ & $c$ & - & - & -
\end{tabular}

where $a:=a_{1}^{-1} a_{2}=b_{2} b_{1}^{-1}, b:=a_{1}^{-1} a_{3}=b_{3} b_{1}^{-1}, c:=$ $a_{1}^{-1} a_{4}$, and $d:=b_{4} b_{1}^{-1}$ and where it is row 4 and column 3 or 4 that have only two of the elements $1, a, b$.

Assume $a^{2} \notin\{1, a, b\}$. Since row 2 and column 2 both contains $1, a, b$, we have $b a=1, c a=b$, and $a b=1$ and $a d$ $=b$. This implies $b=a^{-1}$ and $c=d=a^{-2}$. Since $1, a, b, c$ are distinct $a$ has order at least four. Use that row 3 has all 
three elements $1, a, a^{-1}$ to conclude that $a$ has order 4 and, therefore, 4 divides $n_{1}$.

Assume $b a \notin\{1, a, b\}$; then $a^{2}=1$ or $a^{2}=b$. If $a^{2}=1$ then $c a=b$ and therefore $c=b a$. Since row 2 contains $1, a$, $b$, we get $a d=b$ and therefore $d=a b$. The same argument is applied to row 3 gives $b^{2}=1$ and $b d=a$ or $b^{2}=a$ and $b d$ $=1$. If $b^{2}=a$ then $b$ is an element of order 4 . If $b^{2}=1$ then $d=b^{2} d=b(b d)=b a=c$ and then $\{1, a, b, c\}=\{1, a$, $b, d\}$ is the Klein Four Group.

If $a^{2}=b$ then $b a=1$ or $c a=1 . b a=1$ implies $a^{3}=1$ and $b^{2}=a$ and makes it impossible for row 4 and column 4 to contain more than one of the elements $1, a, b$. Therefore, $c a$ $=1$ and in row 2 , we get $a d=1$. Then $c=a^{-1}=d$ and $\{1$, $a, b, c\}=\{1, a, b, d\}=\left\{1, a, a^{2}, a^{-1}\right\}$ and it follows as before that $a$ has order 4 .

Assume ca $\notin\{1, a, b\}$. Look at column 2 and conclude that $b a=1$ and therefore $a^{2}=b$. Row 3 implies that $b^{2}=a$ or $b d=a \cdot b^{2}=a$ contradicts the assumption that row 4 and column 4 contains two of the elements $1, a, b$. Therefore, $b d$ $=a$. Since $1=b a=a b$, it must be column 3 that contains all the elements $1, a, b$ and by looking at row 3 , we get that $c b=$ $a$ which implies $c=a^{2}$. Multiply $b d=a$ by $a$ from the left and get $d=a^{2}=c$. We now have the elements $1, a, a^{2}, a^{-1}$ and as before, we conclude that $a$ has order 4 .

Case 2): Assume that we have 11 inlet channels appearing on four switches in block 1 , on three switches in block 2 , and on four switches in block 3 . In the induced latin square, we can find 11 entries appearing in four rows and four columns so that the 11 entries contains only four different elements. By appropriate multiplication as in the former case, we may assume that the row elements are $1, a, b, c$ and the column elements are $1, a, b$. Since only four different elements are present in the 11 entries, we have $C_{1}=C_{2}$ or $C_{1}=C_{3}$. and $\left|C_{1} \cap C_{j}\right| \geq 3$ for $j=2,3$. We assume that $C_{1}=C_{2}$. If $a^{2}=$ 1 , we conclude by lemma A.1 that 4 divides $n_{1}$. If $a^{2}=b$, then $b a=1$ or $b a=c$. Since $b a=1$ forces $c a=c$, we conclude that $b a=c$. Then $c a=1$ yielding $1=b a^{2}=a^{4}$ and $c=a^{3}$. Therefore, $a$ is an element of order 4 and 4 divides $n_{1}$. When $a^{2}=c$ the same argument yields that $a$ has order 4 .

$n=13$ : Assume $S_{3, n_{1}}(13)=12$. If the 13 inlet channels appeared on only three switches in one of the blocks this would force the inlet channels to appear on at least five switches in each of the remaining blocks. We may, therefore, assume that in the induced latin square, there exist 13 entries containing only four different elements $x_{1}, x_{2}, x_{3}, x_{4}$ and appearing in four rows and four columns. There exists at least one row and one column each containing all the elements $x_{1}, x_{2}, x_{3}, x_{4}$. By appropriate multiplication as in Case 1) of $n=11$, we may assume that the four rows and the four columns correspond to the elements $1, a, b, c$. If there exist two columns both containing these four elements, we can proceed exactly as in Case 2) of $n=11$ and conclude that 4 divides $n_{1}$. We may, therefore, assume that $\left|C_{1} \cap C_{j}\right|=3$ for $j=2,3,4$. From this we see that if $a^{2}=1$ then by lemma A.1, we conclude that 4 divides $n_{1}$.

\begin{tabular}{c|cccc} 
& 1 & $a$ & $b$ & $c$ \\
\hline 1 & 1 & $a$ & $b$ & $c$ \\
$a$ & $a$ & 1 & - & - \\
$b$ & $b$ & - & - & - \\
$c$ & $c$ & - & - & -
\end{tabular}.

Assume that $a^{2}$ is unknown, i.e., we do not know whether or not $a^{2} \in C_{1}$. Then $b a=1$ or $b a=c$. If $b a=1$ then $c a=$ $b$. Then we see $b=a^{-1}$ and $c=a^{-2}$. Therefore, we only have to consider the multiplication table of $1, a, a^{-1}, a^{-2}$. Since $\left|C_{1} \cap C_{4}\right|=3$, we get $a^{-3} \in\{1, a\}$ or $a^{-4} \in\{1, a\}$ yielding $a$ has order 4 or 5 . The conditions $\left|C_{1} \cap C_{j}\right|=3$ for $j=2,4$ give no further information.
If $b a=c$ then $c a=b$ or $c a=1 . c a=b$ implies $c a^{2}=c$ implying $a^{2}=1$ and by lemma A.1, we conclude that 4 divides $n_{1}, c a=1$ implies $c=a^{-1}$ and $b^{2} a=1$ and we have once more the multiplication table of $1, a, a^{-1}, a^{-2}$ from which we conclude that 4 or 5 divides $n_{1}$.

Assume that $a b$ is unknown. Then $a^{2}=1$ or $a^{2}=c$. If $a^{2}$ $=1$ lemma A.1 yields that 4 divides $n_{1}$. If $a^{2}=c$ then $c a=1$ or $c a=b$. $c a=1$ implies $a^{3}=1$ and by using $\left|C_{1} \cap C_{3}\right|=$ 3 this implies $b=c$ or column 3 contains the same element twice; in both cases a contradiction. Therefore, $c a=b$ implying $b=a^{3}$, i.e., $\{1, a, b, c\}=\left\{1, a, a^{2}, a^{3}\right\}$. As in the case $a^{2}$ unknown, this yields 4 divides $n_{1}$ or 5 divides $n_{1}$.

Since $a c$ unknown proceeds the same way and gives the same result, it will be omitted. The calculation of $S_{3, n_{1}}(13)$ is therefore completed.

\section{APPENDIX B}

\section{A LOWER ESTIMATE FOR $S_{M, n_{1}}$}

In this Appendix, a proof of Result 4.1 is given. The $M$ blocks in the first stage is denoted by $B_{1}, B_{2}, \cdots, B_{M}$ and the $M n_{1}$ first-stage switches are denoted $I_{1}, \cdots, I_{M n_{1}}$.

Let $E$ be of subset of the set of inlet channels, assume that $E$ has $n$ elements and put

$k_{i}(E):=$ The number of switches in $B_{i}$ having elements

from $E$ among their inlet channels.

$$
k(E):=\min \left\{k_{i}(E) \mid i=1,2, \cdots, M\right\} .
$$

$a_{j}(E):=$ The number of elements from $E$ appearing on $I_{j}$.

$a(E):=\max \left\{a_{j}(E) \mid j=1,2, \cdots, M n_{1}\right\}$.

If $x$ is a real number $\lceil x\rceil$ denoted the smallest integer not less than $x$. In this notation, a switch exist, which has at least $\lceil n /$ $k(E)]$ elements from $E$ among its inlet channels. Therefore,

$$
a(E) \geq\lceil n / k(E)\rceil .
$$

The definition of $k(E)$ and $T_{M, n_{1}}(2.1)$ ensures

$$
T_{M, n_{1}}(E) \geq k(E) M \text {. }
$$

The pair condition gives

$$
T_{M, n_{1}}(E) \geq a(E)(M-1)+k(E) .
$$

From 2.2, B.1, B.2, and B.3, we get

$$
\begin{aligned}
S_{M, n_{1}}(n) \geq \min \left\{\operatorname { m a x } \left[k(E) M,\left[\frac{n}{k(E)}\right]\right.\right. & \cdot(M-1)+k(E)] \mid E \text { has } n \text { elements }\} .
\end{aligned}
$$

When $E$ runs through all subsets with $n$ elements, $k(E)$ runs through a subset of $\left\{1,2, \cdots n_{1}\right\}$ yielding

$$
\begin{aligned}
S_{M, n_{1}}(n) \geq \min & \left\{\operatorname { m a x } \left[k M,\left\lceil\frac{n}{k}\right\rceil\right.\right. \\
& \left.\cdot(M-1)+k] \mid k=1,2, \cdots, n_{1}\right\} .
\end{aligned}
$$

Put $f_{1}(k):=k M$ and $f_{2, n}(k):=\lceil n / k\rceil(M-1)+k$. To finish the proof of Result 4.1 , it is enough to show that

$$
\min _{k}\left\{\max \left[f_{1}(k), f_{2, n}(k)\right]\right\}=G_{M}(n) \quad \text { for any } n \leq n_{1}^{2} .
$$

Choose $p$ as the integer having the property

$$
n=p^{2}+x \text { where } 1 \leq x \leq 2 p+1 .
$$


First assume that $1 \leq x \leq p$. Then for $k=0,1, \cdots, p-1$, we have

$$
\begin{aligned}
f_{2, n}(p-k) & =\left\lceil\frac{p^{2}+x}{p-k}\right\rceil(M-1)+p-k \\
& \geq(p+k+1)(M-1)+p-k \\
& =(p+1) M+k(M-2)-1 \\
& \geq(p+1) M-1 .
\end{aligned}
$$

Since $f_{2, n}(p)=(p+1) M-1$ and $f_{1}(k) \geq(p+1) M$ for $k$ $\geq p+1$, it is now proved that

$$
\begin{aligned}
\min _{k}\left\{\max \left[f_{1}(k), f_{2, n}(k)\right]\right\}=(p+1) M-1 \\
\text { when } n=p^{2}+x \text { and } 1 \leq x \leq p .
\end{aligned}
$$

Assume now that $p+1 \leq x \leq 2 p+1$. Then for $k=0,1$, $\cdots, p-1$ we have

$$
\begin{aligned}
f_{2, n}(p-k) & \\
& =\left\lceil\frac{(p-k)(p+k+1)+k^{2}+k+x-p}{p-k}\right](M-1)+p-k \\
& \geq(p+k+2)(M-1)+p-k \\
& =(p+1) M+(k+1)(M-2) \\
& \geq(p+1) M .
\end{aligned}
$$

Since $f_{2, n}(p+1)=(p+1) M$ and $f_{1}(k) \geq(p+1) M$ for $k$ $\geq p+1$ it is proved that

$$
\begin{aligned}
\min _{k}\left\{\max \left[f_{1}(k), f_{2, n}(k)\right]\right\}=(p+1) M \\
\text { when } n=p^{2}+x \text { and } p+1 \leq x \leq 2 p+1 .
\end{aligned}
$$

B.5, B.7, and B.8 concludes the proof of Result 4.1 .

\section{APPENDIX C}

\section{Proof of Result 4.3}

In this Appendix, we prove Result 4.3. It is enough to show if $k \geq 3+1 /(\ln 2 \ln )\left(M^{2} \ln M\right)$ then $g_{k} \geq M^{2}$. The following lemma will be helpful.

Lemma C.1: For $k \geq 2$ and $M \geq 2$ the following estimate is valid:

$$
g_{k} \geq M^{2-2^{1-k}}\left[1+\left(\sum_{i=0}^{k-2} 3^{i}\right) 3^{1-k} M^{-2}\right] .
$$

Proof: We have $g_{2}=G_{M}(M+1) \geq M(M+1)^{1 / 2} \geq$ $M^{3 / 2}(1+1 / 3 M) \geq M^{3 / 2}\left(1+1 / 3 M^{2}\right)$. This proves the lemma for $k=2$. Assume the lemma is true for some $k$. Then,

$$
\begin{aligned}
g_{k+1}=G_{M}\left(g_{k}+1\right) \geq & M\left(g_{k}+1\right)^{1 / 2} \\
\geq & M\left(M ^ { 2 - 2 ^ { 1 - k } } \left(1+\left(\sum_{i=0}^{k-2} 3^{i}\right)\right.\right. \\
& \left.\left.\cdot 3^{1-k} M^{-2}\right)+1\right)^{1 / 2} \\
= & M^{2-2^{k}}\left(1+\left(\sum_{i=0}^{k-2} 3^{i}\right)\right. \\
& \left.\cdot 3^{1-k} M^{-2}+M^{2^{1 / 2-k}-2}\right)
\end{aligned}
$$

$$
\begin{aligned}
\geq & M^{2-2^{-k}}\left(1+\left(\sum_{i=0}^{k-2} 3^{i}\right)\right. \\
& \left.\cdot 3^{-k} M^{-2}+\frac{1}{3} M^{-2}\right) \\
= & M^{2-2^{1-(k+1)}}\left(1+\left(\sum_{i=0}^{k-1} 3^{i}\right)\right. \\
& \left.\cdot 3^{1-(k+1)} M^{-2}\right)
\end{aligned}
$$

which concludes the proof of the lemma.

Now assume $k \geq 3+(1 / \ln 2) \ln \left(M^{2} \ln M\right)$. By taken exp, we obtain

$$
2^{k^{\prime}-3} \geq M^{2} \ln M
$$

Since $k \geq 2$, we have $\left(3^{k-1}-1\right) / 3^{k-1} \geq 2 / 3$ yielding

$2^{k-3} \frac{3}{2} \frac{3^{k-1}-1}{3^{k-1}} \geq M^{2} \ln M \Leftrightarrow \frac{3}{4} \frac{3^{k-1}-1}{2 \cdot 3^{k-1}} M^{-2} \geq 2^{1-k} \ln M$.

Since $\ln (1+x) \geq(3 / 4) x$ for $x$ small, this implies

$$
\ln \left[1+\frac{3^{k-1}-1}{2 \cdot 3^{k-1}} M^{-2}\right] \geq 2^{1-k} \ln M \text {. }
$$

Take exp and multiply by $M^{2-2^{1-k}}$ and obtain

$$
M^{2-2^{1-k}}\left(1+\frac{3^{k-1}-1}{2 \cdot 3^{k-1}} M^{-2}\right) \geq M^{2}
$$

But,

$$
\begin{aligned}
& M^{2-2^{1-k}}\left(1+\frac{3^{k-1}-1}{2 \cdot 3^{k-1}} M^{-2}\right) \\
& \quad=M^{2-2^{1-k}}\left(1+\left(\sum_{i=0}^{k-2} 3^{i}\right) 3^{1-k} M^{-2}\right) \\
& =g_{k} .
\end{aligned}
$$

and the proof is completed.

\section{REFERENCES}

[1] G. W. Richards and F. K. Hwang, "A two-stage rearrangeable broadcast switching network," IEEE Trans. Commun., vol. COM33, pp. 1025-1035, 1985.

[2] P. Hall, "On representatives of subsets," J. London Math. Soc., vol. 10, pp. 26-30, 1935.

[3] F. K. Hwang and G. W. Richards, "On the capacity of the subarray partial concentrator," preprint.

[4] J. Denes and A. D. Keedwell, Latin Squares and their Applications. English Universities Press, 1974.

[5] P. Dembowski, Finite Geometries. New York: Springer-Verlag, 1968.

[6] S. Lang, Algebra. Reading, MA: Addison-Wesley, 1984.

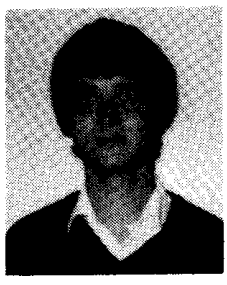

Sqren B. Jacobsen received the M.Sc. in mathematics from Aarhus University, Denmark, in January 1986.

During 1986, he was employed at Electromagnetics Institute, Technical University of Denmark. In 1987 he was temporarily with the Mathematics Departments at UCLA and M.I.T. He returned to Electromagnetics Institute, Technical University of Denmark in January 1988. His interests include switching networks, combinatorics, ATD, and queueing systems. 